\title{
Teaching of biochemistry: analyze of works presented in Congress the Society Brazilian Biochemistry and Molecular Biology - SBBq
}

Escoto, D.F.. ${ }^{1}$; Pereira, G.C. ${ }^{1}$; Soares C.B. ${ }^{1}$; Dos Santos, M..E.T. ${ }^{2}$; Folmer, V. ${ }^{3}$

${ }^{1}$ Licenciatura em Ciências da Natureza, Campus Uruguaiana, Universidade Federal do Pampa, Rio Grande do Sul, Brasil; ${ }^{3}$ PPG Educação em Ciências: Química da vida e Saúde, Universidade Federal de Santa Maria, Rio Grande do Sul, Brasil; ${ }^{3}$ PPG Bioquímica, Campus Uruguaiana, Universidade Federal do Pampa, Rio Grande do Sul, Brasil

Introduction: In recent decades the strategies to improve science education has grown exponentially. Thus, the scientific production in the area is also growing, with the purpose of identifying parameters and methodologies that contribute to their qualification. The teaching of biochemistry is intimately linked to that context. However, it is still little explored in basic education and with character technicist in higher education. The aim of this study was identify areas that received most attention in the scientific literature about teaching and education in biochemistry that were presented at the Congress of the Brazilian Society of Biochemistry and Molecular Biology from 2004 to 2012. Material and Methods: To conduct the survey were analyzed summaries available on the website of the Brazilian Journal of Education for Biochemistry and Molecular Biology published in the proceedings of the event, where they were encontrados176 summaries. For expression of results was used categorization from the content analysis. Results and Discussion: The results observed to establish nine categories based on the analysis of the titles and content of the work, which, in descending order, were: information and communication technologies, alternative methods teaching and learning, biochemistry in Elementary Education and / or Medium, experiential activities, teacher training, dissemination of science, proposition and evaluation of content and / or science curricular and History and Philosophy. It is noticed that the three most important categories were consolidated along editions. In education, however, there was a significant decrease in the number of abstracts submitted abstracts for the past five years. Conclusions: We conclude that all categories listed seeking alternatives to improve teaching practices and promote education of biochemistry in different contexts.

Key-words: teaching biochemistry, content analysis, science education. Supported by: CAPES 